\title{
Synthesis of naphthoxazinone derivatives using silica-bonded $S$-sulfonic acid as catalyst under solvent-free conditions
}

\author{
KHODABAKHSH NIKNAM* and PARISA ABOLPOUR \\ Department of Chemistry, Faculty of Sciences, Persian Gulf University, Bushehr 75169 Iran \\ e-mail: niknam@pgu.ac.ir; khniknam@gmail.com
}

MS received 19 September 2014; revised 17 April 2015; accepted 17 April 2015

\begin{abstract}
Silica-bonded $S$-sulfonic acid is employed as a recyclable catalyst for the synthesis of naphthoxazinone derivatives from the reaction of $\beta$-naphthol, aromatic aldehydes and urea at $150^{\circ} \mathrm{C}$ under solvent-free conditions. The heterogeneous catalyst was recycled for five runs after the reaction of $\beta$-naphthol, benzaldehyde and urea without losing its catalytic activity.
\end{abstract}

Keywords. Silica-bonded propyl-S-sulfonic acid; naphthoxazinones; aromatic aldehydes; heterogeneous catalysts; solid acids.

\section{Introduction}

The important advantages of solid acids and bases such as operational simplicity, environmental compatibility, non-toxicity, reusability, low cost, and ease of isolation recommend them as suitable catalytic systems in organic synthesis. ${ }^{1-4}$ In fact, simplified recovery and reusability are critical advantages of heterogeneous catalytic systems, which could lead to novel environmentally benign chemical procedures for academia and industry. ${ }^{1-5}$ Metal colloids, mineral clays and supported reagents on silica gel, alumina and other solid supports are various types of heterogeneous and reusable catalytic systems, which have been designed and used in organic synthesis. Among them, silica-supported catalysts have attracted more attention because they are inexpensive, easy to prepare, and insoluble in most of organic solvents, which make them being recycled from various reactions

Along the line of our studies in preparation and application of solid acid and base catalysts in chemical transformations, ${ }^{6-24}$ herein, we report the catalytic activity of some of these catalysts such as, silica-bonded propyl $S$-sulfonic acid (1), silica-bonded $N$-propyl sulfamic acid (2), silica-bonded tin chloride (3), silicabonded titanium chloride (4), silica-bonded n-propylimidazolium hydrogen sulfate (5), and silica-bonded n-propyl-methylimidazolium hydrogen sulfate (6)] as heterogeneous solid acid for the synthesis of naphthaoxazines (scheme 1).

Aromatic-condensed oxazinone derivatives are an important class of heterocyclic compounds, since

\footnotetext{
*For correspondence
}

many of these heterocyclic systems exhibit biological activities. ${ }^{25}$ Among them, naphthalene-condensed 1,3oxazin-3-ones have been reported to act as antibacterial agents. ${ }^{25,26}$ This class of compounds has also been used as precursors in the preparation of phosphinic ligands for asymmetric catalysis. ${ }^{27}$ Due to the pharmacological importance, several protocols have been developed over a period of time. The conventional method includes condensation of phenols, formaldehyde and amines through Mannich reaction. ${ }^{28}$ Additionally they have been synthesized by the reaction of amino alkylnaphthols with phosgene ${ }^{29}$ and reaction with carbonyl di-imidazole. ${ }^{30}$ However, these methods have some drawbacks like prolonged reaction time, lack of easy availability/ preparation of starting materials, and hazardous reaction conditions.

Recently, the preparation of naphthoxazinone derivatives has been achieved in the presence of various catalysts such as $p$-TSA, ${ }^{31}[\mathrm{bmim}] \mathrm{Br},{ }^{32} \mathrm{TMSCl} / \mathrm{NaI},{ }^{33}$ $\mathrm{HClO}_{4} / \mathrm{SiO}_{2},{ }^{34} \mathrm{TMSCl},{ }^{35}$ silica gel, ${ }^{36}$ phosphomolybdic acid, ${ }^{37} \mathrm{Cu}$ NPs, ${ }^{38}$ Thiamine hydrochloride, ${ }^{39} \mathrm{ZnO}$ $\mathrm{NPs},{ }^{40} \mathrm{FeCl}_{3} / \mathrm{SiO}_{2} \mathrm{NPs},{ }^{41}$ and silica supported Preyssler heteropoly acid. ${ }^{42}$

\section{Experimental}

\subsection{General}

Chemicals were purchased from Fluka, Merck and Aldrich Chemical Companies. All the products were characterized by comparison of their IR, ${ }^{1} \mathrm{H}$ NMR and ${ }^{13} \mathrm{C}$ NMR spectroscopic data and their melting points 


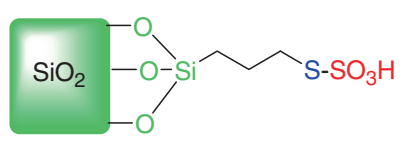

1

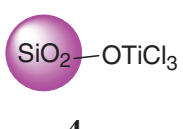

4

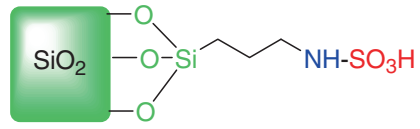

2

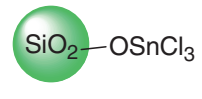

3

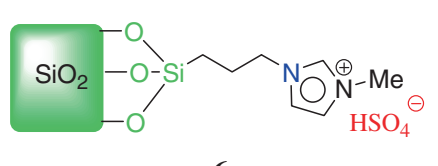

6

Scheme 1. The structure of silica functionalized catalysts which were used.

with reported values. ${ }^{31-42}$ Solid acids 1-6 were prepared according to our previous reported methods. ${ }^{6-8,12,19,23,24}$

\subsection{General procedure for the synthesis of naphthoxazinones}

A mixture of $\beta$-naphthol ( $1 \mathrm{mmol})$, aromatic aldehyde (1 mmol), urea (1.2 mmol), 1 (0.02 g, $0.68 \mathrm{~mol} \%)$, under solvent-free conditions was heated at $150^{\circ} \mathrm{C}$ for the time specified in table 2 . The reaction was followed by TLC. After completion of the reaction, ethanol $(10 \mathrm{~mL})$ was added and filtered. The remaining solid was washed with warm ethanol $(3 \times 5 \mathrm{~mL})$ in order to separate catalyst. After cooling the organic phase, the crude product was precipitated and filtered. The crude sample was passed through a short column chromatography using n-hexane/ ethyl acetate as eluent. The recovered catalyst was dried and reused for subsequent runs.

2.2a 1,2-Dihydro-1-(phenyl)naphtho[1,2-e][1,3]oxazine-3-one (4a): M.p. $220-222^{\circ} \mathrm{C}$, (Lit. ${ }^{31}$ M.p. $218-220^{\circ} \mathrm{C}$ ); ${ }^{1} \mathrm{H}$ NMR (400 MHz, DMSO-d $\left.{ }_{6}\right): \delta(\mathrm{ppm}) 6.20(\mathrm{~d}, 1 \mathrm{H}$, $J=2.7 \mathrm{~Hz}, \mathrm{CH}), 7.23-7.35$ (m, 5H, Ar), 7.39 (d, $1 \mathrm{H}$, $J=7.1 \mathrm{~Hz}, \mathrm{Ar}), 7.43-7.51(\mathrm{~m}, 2 \mathrm{H}, \mathrm{Ar}), 7.82(\mathrm{~d}, 1 \mathrm{H}$, $J=6.6 \mathrm{~Hz}, \operatorname{Ar}), 7.95(\mathrm{~d}, 1 \mathrm{H}, J=7.3 \mathrm{~Hz}, \mathrm{Ar}), 8.00$ $(\mathrm{d}, 1 \mathrm{H}, J=7.3 \mathrm{~Hz}, \mathrm{Ar}), 8.86(\mathrm{~d}, 1 \mathrm{H}, J=2.2 \mathrm{~Hz}$, $\mathrm{NH}) ;{ }^{13} \mathrm{C}$ NMR (100 MHz, DMSO-d 6 ): $\delta(\mathrm{ppm})$ 54.23, 114.52, 117.33, 123.58, 125.55, 127.43, 127.83, $128.48,129.09,129.35,129.41,130.69,130.88$, $143.34,147.89,149.77$.

2.2b 1,2-Dihydro-1-(4-chlorophenyl)naphtho[1,2-e] [1,3]oxazine-3-one (4b): M.p. 208-210 ${ }^{\circ}$ C, (Lit. ${ }^{34}$ M.p. 212-214 ${ }^{\circ} \mathrm{C}$ ); ${ }^{1} \mathrm{H}$ NMR (400 MHz, DMSO-d ${ }_{6}$ ): $\delta$ (ppm) $6.25(\mathrm{~s}, 1 \mathrm{H}, \mathrm{CH}), 7.32-7.35(\mathrm{~m}, 2 \mathrm{H}, \mathrm{Ar}), 7.38-7.41$ $(\mathrm{m}, 3 \mathrm{H}, \mathrm{Ar}), 7.44-7.51(\mathrm{~m}, 2 \mathrm{H}, \mathrm{Ar}), 7.79(\mathrm{~d}, 1 \mathrm{H}$, $J=6.3 \mathrm{~Hz}, \operatorname{Ar}), 7.96(\mathrm{~d}, 1 \mathrm{H}, J=6.3 \mathrm{~Hz}, \mathrm{Ar})$, $8.01(\mathrm{~d}, 1 \mathrm{H}, J=7.1 \mathrm{~Hz}, \operatorname{Ar}), 8.91(\mathrm{~s}, 1 \mathrm{H}, \mathrm{NH})$; ${ }^{13} \mathrm{C}$ NMR (100 MHz, DMSO-d 6 ): $\delta$ (ppm) 53.43, $114.04,117.36,123.51,125.63,127.93,129.14$, $129.38,129.43,130.89,142.24,147.92,149.62$. 2.2c 1,2-Dihydro-1-(4-bromophenyl)naphtho[1,2-e] [1,3]oxazine-3-one (4c): M.p. 218-220 ${ }^{\circ}$, (Lit. ${ }^{38}$ M.p. $\left.218-219^{\circ} \mathrm{C}\right) ;{ }^{1} \mathrm{H}$ NMR $\left(400 \mathrm{MHz}, \mathrm{DMSO}-\mathrm{d}_{6}\right): \delta(\mathrm{ppm})$ $6.23(\mathrm{~d}, 1 \mathrm{H}, J=2.4 \mathrm{~Hz}, \mathrm{CH}), 7.27\left(\mathrm{dt}, 2 \mathrm{H}, J_{1}=6.8 \mathrm{~Hz}\right.$, $\left.J_{2}=1.8 \mathrm{~Hz}, \mathrm{Ar}\right), 7.38(\mathrm{~d}, 1 \mathrm{H}, J=7.3 \mathrm{~Hz}, \mathrm{Ar}), 7.44-7.51$ $(\mathrm{m}, 2 \mathrm{H}, \mathrm{Ar}), 7.54\left(\mathrm{dt}, 2 \mathrm{H}, J_{1}=6.8 \mathrm{~Hz}, J_{2}=1.8 \mathrm{~Hz}\right.$, $\operatorname{Ar}), 7.79(\mathrm{~d}, 1 \mathrm{H}, J=6.6 \mathrm{~Hz}, \mathrm{Ar}), 7.96(\mathrm{~d}, 1 \mathrm{H}, J=7.3 \mathrm{~Hz}$, Ar), $8.01(\mathrm{~d}, 1 \mathrm{H}, J=7.3 \mathrm{~Hz}, \mathrm{Ar}), 8.90(\mathrm{~d}, 1 \mathrm{H}, J=2.4 \mathrm{~Hz}$, $\mathrm{NH}) ;{ }^{13} \mathrm{C}$ NMR (100 MHz, DMSO-d 6 ): $\delta$ (ppm) 53.53, 113.97, 117.35, 121.66, 123.50, 125.64, 127.93, 129.15, $129.25,129.71,130.90,132.36,142.64,147.93,149.61$.

2.2d 1,2-Dihydro-1-(4-fluorophenyl)naphtho[1,2-e][1, 3]oxazine-3-one $(\mathbf{4 d})$ : M.p. 200-202 ${ }^{\circ}$ C, (Lit. ${ }^{42}$ M.p. 201-203 $\left.{ }^{\circ} \mathrm{C}\right) ;{ }^{1} \mathrm{H}$ NMR $\left(400 \mathrm{MHz}\right.$, DMSO-d $\left.{ }_{6}\right): \delta(\mathrm{ppm})$ $6.25(\mathrm{~s}, 1 \mathrm{H}, \mathrm{CH}), 7.16(\mathrm{t}, 2 \mathrm{H}, J=6.8 \mathrm{~Hz}, \mathrm{Ar}), 7.34-$ 7.40 (m, 3H, Ar), 7.44-7.53 (m, 2H, Ar), 7.80 (d, 1H, $J=6.4 \mathrm{~Hz}, \mathrm{Ar}), 7.96(\mathrm{~d}, 1 \mathrm{H}, J=6.1 \mathrm{~Hz}, \mathrm{Ar}), 8.00$ $(\mathrm{d}, 1 \mathrm{H}, J=7.1 \mathrm{~Hz}, \mathrm{Ar}), 8.89(\mathrm{~s}, 1 \mathrm{H}, \mathrm{NH}) ;{ }^{13} \mathrm{C}$ NMR $\left(100 \mathrm{MHz}, \mathrm{DMSO}-\mathrm{d}_{6}\right): \delta$ (ppm) 53.39, 114.32, 116.14, $116.32,117.36,123.54,125.60,127.89,129.13,129.26$, $129.54,129.61,130.82,130.89,147.86,149.68,162.05$ $\left(\mathrm{d}, J_{C-F}=194.3 \mathrm{~Hz}\right)$.

2.2e 1,2-Dihydro-1-(3-fluorophenyl)naphtho[1,2-e][1, 3]oxazine-3-one $(4 e)$ : M.p. $217-219^{\circ} \mathrm{C}$, (Lit. ${ }^{40}$ M.p. 218-220 $\left.{ }^{\circ} \mathrm{C}\right) ;{ }^{1} \mathrm{H}$ NMR (400 MHz, DMSO-d 6 ): $\delta(\mathrm{ppm})$ $6.26(\mathrm{~d}, 1 \mathrm{H}, J=2.4 \mathrm{~Hz}, \mathrm{CH}), 7.06-7.14(\mathrm{~m}, 2 \mathrm{H}, \mathrm{Ar})$, $7.21\left(\mathrm{dt}, 1 \mathrm{H}, J_{1}=7.3 \mathrm{~Hz}, J_{2}=1.7 \mathrm{~Hz}, \mathrm{Ar}\right), 7.35-7.41$ (m, 2H, Ar), 7.45-7.54 (m, 2H, Ar), 7.83 (d, 1H, J = 6.8 $\mathrm{Hz}, \mathrm{Ar}), 7.97\left(\mathrm{dd}, 1 \mathrm{H}, J_{1}=6.6 \mathrm{~Hz}, J_{2}=1.0 \mathrm{~Hz}, \mathrm{Ar}\right)$, $8.02(\mathrm{~d}, 1 \mathrm{H}, J=7.3 \mathrm{~Hz}, \mathrm{Ar}), 8.92(\mathrm{~d}, 1 \mathrm{H}, J=2.4 \mathrm{~Hz}$, $\mathrm{NH}) ;{ }^{13} \mathrm{C}$ NMR (100 MHz, DMSO-d 6 ): $\delta$ (ppm) 53.58, 113.90, 114.37, 114.54, 115.28, 115.45, 117.36, 123.35, 123.52, 125.66, 127.98, 129.14, 129.31, 130.88, 130.94, $131.59,131.65,145.94,145.99,147.99,149.64,162.62$ $\left(\mathrm{d}, \mathrm{J}_{\mathrm{C}-\mathrm{F}}=194.3 \mathrm{~Hz}\right)$.

$2.2 \mathrm{f}$ 1,2-Dihydro-1-(3-nitrophenyl)naphtho[1,2-e][1,3] oxazine-3-one $(4 f)$ : M.p. $210-212{ }^{\circ} \mathrm{C} ;{ }^{1} \mathrm{H}$ NMR $(400 \mathrm{MHz}$, DMSO- $\left.\mathrm{d}_{6}\right): \delta(\mathrm{ppm}) 6.48(\mathrm{~s}, 1 \mathrm{H}, \mathrm{CH}), 7.41-7.54(\mathrm{~m}, 3 \mathrm{H}$, 
Ar), 7.61-7.68 (m, 2H, Ar), 7.85 (d, 1H, $J=6.6 \mathrm{~Hz}, \mathrm{Ar})$, $7.98(\mathrm{~d}, 1 \mathrm{H}, J=6.3 \mathrm{~Hz}, \mathrm{Ar}), 8.05(\mathrm{~d}, 1 \mathrm{H}, J=7.3 \mathrm{~Hz}$, Ar), 8.15 (d, 1H, $J=7.9 \mathrm{~Hz}, \mathrm{Ar}), 8.29$ (s, 1H, Ar), 9.03 (s, $1 \mathrm{H}, \mathrm{NH}) .{ }^{13} \mathrm{C}$ NMR $\left(100 \mathrm{MHz}, \mathrm{DMSO}-\mathrm{d}_{6}\right): \delta(\mathrm{ppm})$ 52.78, 112.91, 116.91, 121.87, 122.98, 123.08, 125.27, 127.64, 128.72, 130.43, 130.75, 133.45, 144.68, 147.71, 147.98, 149.01. Elemental analysis: for $\mathrm{C}_{18} \mathrm{H}_{12} \mathrm{~N}_{2} \mathrm{O}_{4}$ calculated: C, 67.50; H, 3.78; N, 8.75. Found: C, 67.29; $\mathrm{H}, 3.89 ; \mathrm{N}, 8.59$.

$2.2 \mathrm{~g}$ 1,2-Dihydro-1-(4-methoxyphenyl)naphtho[1,2-e] [1,3]oxazine-3-one $(\mathbf{4 g})$ : M.p. $186-188^{\circ} \mathrm{C}$, (Lit. ${ }^{40} \mathrm{M} . \mathrm{p}$. $\left.188-190^{\circ} \mathrm{C}\right) ;{ }^{1} \mathrm{H}$ NMR $(400 \mathrm{MHz}$, DMSO-d 6 ): $\delta(\mathrm{ppm})$ 3.69 (s, 3H, OMe), 6.14 (d, 1H, $J=2.4 \mathrm{~Hz}, \mathrm{CH}), 6.88$ $\left(\mathrm{dt}, 2 \mathrm{H}, J_{1}=7.1 \mathrm{~Hz}, J_{2}=2.1 \mathrm{~Hz}, \mathrm{Ar}\right), 7.22(\mathrm{dt}, 2 \mathrm{H}$, $\left.J_{1}=7.1 \mathrm{~Hz}, J_{2}=2.1 \mathrm{~Hz}, \mathrm{Ar}\right), 7.37(\mathrm{~d}, 1 \mathrm{H}, J=7.1 \mathrm{~Hz}$, Ar), 7.43-7.51 (m, 2H, Ar), 7.80 (d, 1H, $J=6.6 \mathrm{~Hz}, \mathrm{Ar})$, $7.94(\mathrm{~d}, 1 \mathrm{H}, J=7.6 \mathrm{~Hz}, \mathrm{Ar}), 7.98(\mathrm{~d}, 1 \mathrm{H}, J=7.3 \mathrm{~Hz}$, $\mathrm{Ar}), 8.79(\mathrm{~d}, 1 \mathrm{H}, J=2.4 \mathrm{~Hz}, \mathrm{NH}) ;{ }^{13} \mathrm{C} \mathrm{NMR}(100 \mathrm{MHz}$, DMSO-d $\left.\mathrm{d}_{6}\right): \delta(\mathrm{ppm}) 53.65,55.56,114.70,114.77,117.32$, 123.62, 125.49, 127.75, 128.66, 129.06, 129.34, 130.55, $130.87,135.50,147.77,149.80,159.31$.

2.2h 1,2-Dihydro-1-(4-iso-propylphenyl)naphtho[1,2-e] [1,3]oxazine-3-one (4h): M.p. $172-174^{\circ} \mathrm{C}$, (Lit. ${ }^{41}$ M.p. $\left.171-172{ }^{\circ} \mathrm{C}\right) ;{ }^{1} \mathrm{H}$ NMR $\left(400 \mathrm{MHz}, \mathrm{DMSO}_{-} \mathrm{d}_{6}\right): \delta(\mathrm{ppm})$ $1.13(\mathrm{~d}, 3 \mathrm{H}, J=5.5 \mathrm{~Hz}, \mathrm{Me}), 1.14(\mathrm{~d}, 3 \mathrm{H}, J=5.5 \mathrm{~Hz}$, $\mathrm{Me}), 2.81(\mathrm{~h}, 1 \mathrm{H}, J=5.5 \mathrm{~Hz}, \mathrm{CH}), 6.15(\mathrm{~d}, 1 \mathrm{H}, J=$ $2.4 \mathrm{~Hz}, \mathrm{CH}), 7.20$ (d, 2H, J = 6.6 Hz, Ar), 7.23 (d, 2H, $J=6.4 \mathrm{~Hz}, \operatorname{Ar}), 7.38(\mathrm{~d}, 1 \mathrm{H}, J=7.1 \mathrm{~Hz}, \mathrm{Ar})$,
7.43-7.52 (m, 2H, Ar), $7.84(\mathrm{~d}, 1 \mathrm{H}, J=6.8 \mathrm{~Hz}, \mathrm{Ar})$, 7.95 (d, $1 \mathrm{H}, J=7.3 \mathrm{~Hz}, \mathrm{Ar}), 7.99(\mathrm{~d}, 1 \mathrm{H}, J=7.3 \mathrm{~Hz}$, Ar), 8.80 (d, $1 \mathrm{H}, J=2.4 \mathrm{~Hz}, \mathrm{NH}) ;{ }^{13} \mathrm{C}$ NMR $(100 \mathrm{MHz}$, DMSO-d ${ }_{6}$ ): $\delta$ (ppm) 24.18, 24.24, 33.54, 53.94, 114.75, 117.33, 123.59, 125.53, 127.31, 127.36, 127.83, 129.08, 129.36, 130.58, 130.87, 140.87, 147.81, 148.67, 149.86 .

$2.2 \mathrm{i}$ 1,2-Dihydro-1-(4-methylphenyl)naphtho[1,2-e][1, 3]oxazine-3-one (4i): M.p. 167-169 ${ }^{\circ} \mathrm{C}$, (Lit. ${ }^{34}$ M.p. 169 $\left.171{ }^{\circ} \mathrm{C}\right) ;{ }^{1} \mathrm{H}$ NMR $\left(400 \mathrm{MHz}, \mathrm{DMSO}-\mathrm{d}_{6}\right): \delta(\mathrm{ppm}) 2.22$ (s, 3H, Me), $6.14(\mathrm{~s}, 1 \mathrm{H}, \mathrm{CH}), 7.12(\mathrm{~d}, 2 \mathrm{H}, J=6.1 \mathrm{~Hz}$, Ar), $7.19(\mathrm{~d}, 2 \mathrm{H}, J=6.1 \mathrm{~Hz}, \mathrm{Ar}), 7.37(\mathrm{~d}, 1 \mathrm{H}, J=7.1 \mathrm{~Hz}$, Ar), 7.43-7.51 (m, 2H, Ar), $7.80(\mathrm{~d}, 1 \mathrm{H}, J=7.2 \mathrm{~Hz}, \mathrm{Ar})$, $7.94(\mathrm{~d}, 1 \mathrm{H}, J=6.4 \mathrm{~Hz}, \mathrm{Ar}), 7.98(\mathrm{~d}, 1 \mathrm{H}, J=7.1 \mathrm{~Hz}$, Ar), 8.82 (s, $1 \mathrm{H}, \mathrm{NH}) ;{ }^{13} \mathrm{C}$ NMR (100 MHz, DMSO-d 6 ): $\delta(\mathrm{ppm}) 21.09,53.96,114.64,117.31,123.60,125.50$, 127.34, 127.77, 129.06, 129.34, 129.90, 130.59, 130.85, 137.77, 140.47, 147.81, 149.79 .

$2.2 \mathrm{j}$ 1,2-Dihydro-1-(3-methylphenyl)naphtho[1,2-e][1, 3]oxazine-3-one $(4 \mathrm{j})$ : M.p. $206-208^{\circ} \mathrm{C}$, (Lit. ${ }^{41}$ M.p. 206-208 $\left.{ }^{\circ} \mathrm{C}\right) ;{ }^{1} \mathrm{H}$ NMR $\left(400 \mathrm{MHz}, \mathrm{DMSO}_{-} \mathrm{d}_{6}\right): \delta$ (ppm) 2.23 (s, 3H, Me), 6.15 (s, 1H, CH), 7.05-7.13 (m, 2H, Ar), $7.15(\mathrm{~s}, 1 \mathrm{H}, \mathrm{Ar}), 7.21(\mathrm{t}, 1 \mathrm{H}, J=6.8 \mathrm{~Hz}, \mathrm{Ar}), 7.39$ (d, $1 \mathrm{H}, J=7.1 \mathrm{~Hz}, \mathrm{Ar}), 7.43-7.52(\mathrm{~m}, 2 \mathrm{H}, \mathrm{Ar}), 7.81$ (d, $1 \mathrm{H}, J=6.8 \mathrm{~Hz}, \mathrm{Ar}$ ), 7.92-7.96 (m, 1H, Ar), 7.99 (dd, $\left.1 \mathrm{H}, J_{1}=7.2 \mathrm{~Hz}, J_{2}=1.0 \mathrm{~Hz}, \mathrm{Ar}\right), 8.82$ (brs, $1 \mathrm{H}$, $\mathrm{NH}) ;{ }^{13} \mathrm{C}$ NMR (100 MHz, DMSO-d 6 ): $\delta$ (ppm) 21.50, 54.33, 114.50, 117.34, 123.56, 124.58, 125.53, 127.81, $127.89,129.08,129.17,129.31,129.39,130.66$, $130.87,138.61,143.33,147.88,149.79$.

Table 1. The reaction of $\beta$-naphthol, benzaldehyde, and urea, in the presence of solid acids 1-6 under solvent-free conditions.

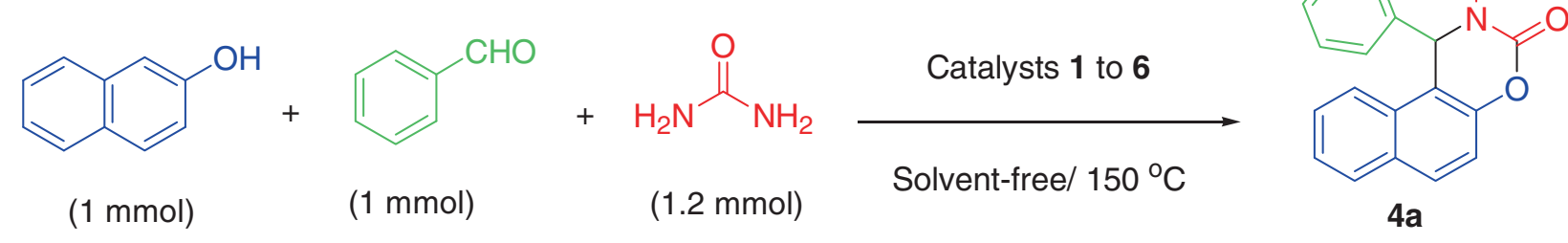

\begin{tabular}{lllll}
\hline Entry & Catalyst & Catalyst loading $(\mathrm{g})$ & Time $(\mathrm{min})$ & ${\text { Yield }{ }^{\mathrm{a}}}^{2}$ \\
1 & No catalyst & - & 720 & trace \\
2 & $\mathbf{1}$ & 0.01 & 90 & 70 \\
3 & $\mathbf{1}$ & 0.02 & 60 & 90 \\
4 & $\mathbf{1}$ & 0.03 & 60 & 90 \\
5 & $\mathbf{2}$ & 0.02 & 60 & 70 \\
6 & $\mathbf{3}$ & 0.02 & 60 & 60 \\
7 & $\mathbf{4}$ & 0.02 & 60 & 50 \\
8 & $\mathbf{5}$ & 0.02 & 60 & 50 \\
9 & $\mathbf{6}$ & 0.02 & 60 & 55 \\
\hline
\end{tabular}

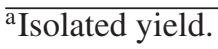




\section{Results and Discussion}

In our initial study, evaluation of a series of silica immobilized acids [silica-bonded propyl $S$-sulfonic acid (1), silica-bonded $N$-propyl sulfamic acid (2), silica supported tin chloride (3), silica supported titanium chloride (4), silica-bonded n-propyl-imidazolium hydrogen sulfate (5), and silica-bonded n-propyl-methylimidazolium hydrogen sulfate (6)] was carried out for the synthesis of naphthoxazinones under thermal and solvent-free conditions. After some preliminary experiments, it was found that a mixture of $\beta$-naphthol, benzaldehyde, and urea at $150^{\circ} \mathrm{C}$ under solvent-free conditions in the presence of a catalytic amount of these heterogeneous solid acids could afford 1,2-dihydro-1-(phenyl)naphtho[1,2-e] [1,3] oxazine-3-ones (4a) in very good yield (table 1).

Table 2. Synthesis of various naphthoxazinones in the presence of $\mathbf{1}$ under solvent-free conditions at $150^{\circ} \mathrm{C}$.

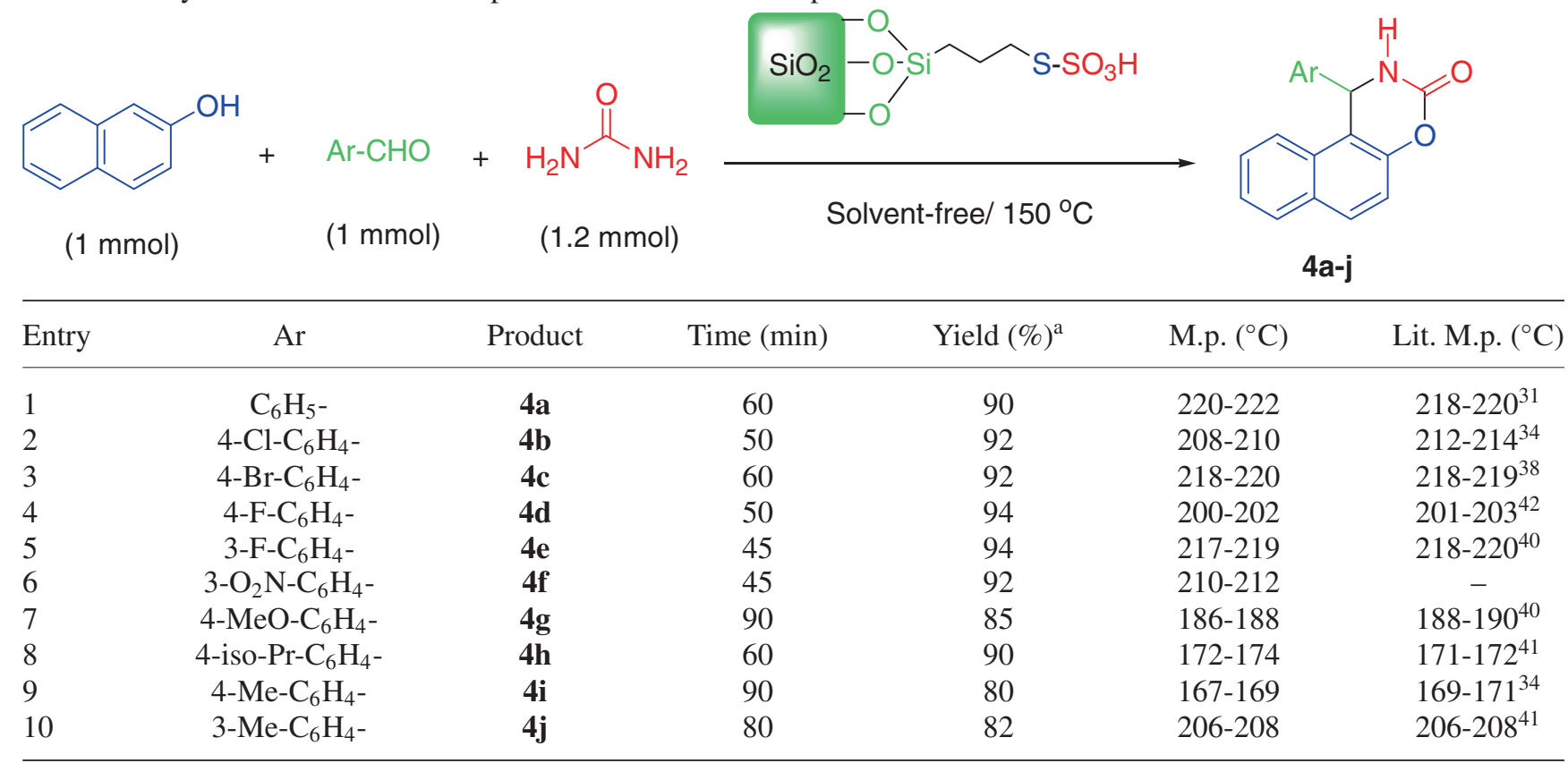

${ }^{\mathrm{a}}$ Isolated yield.

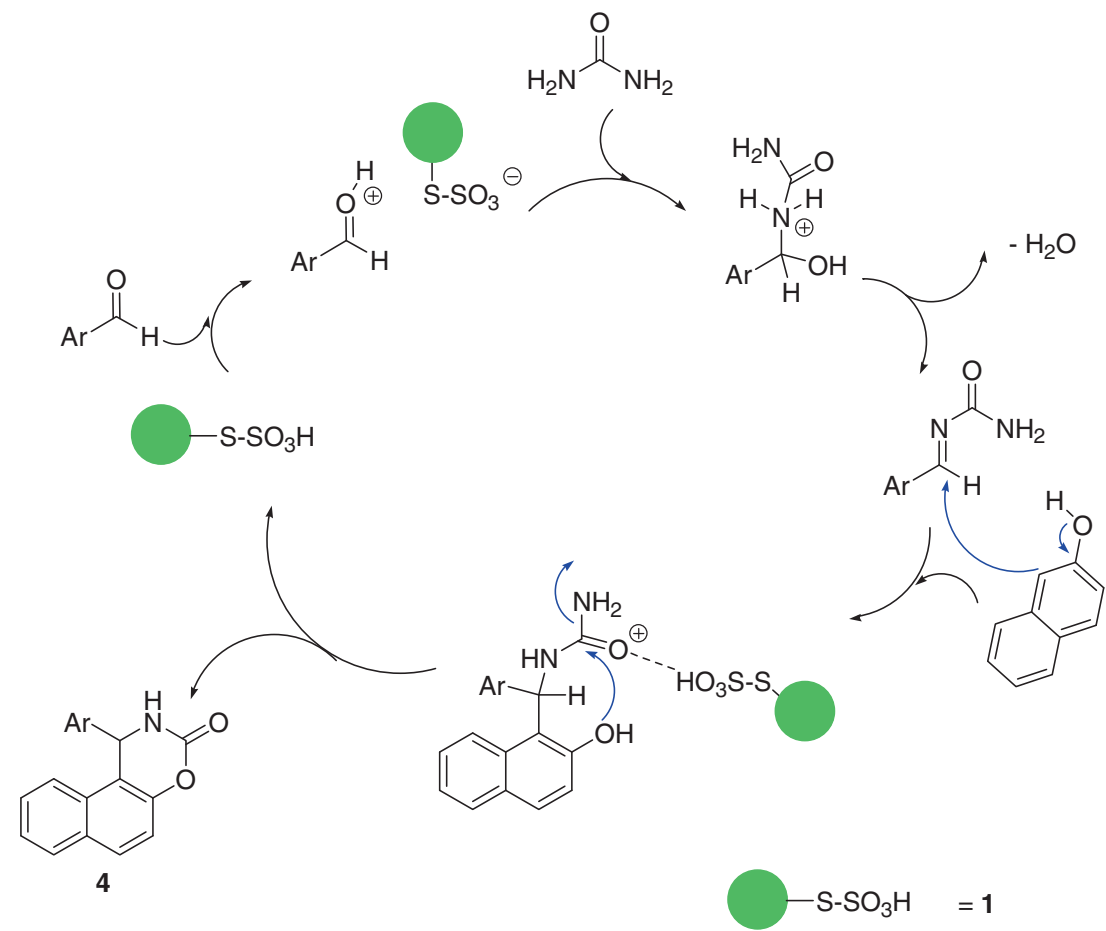

Scheme 2. A plausible mechanism for the synthesis of naphthoxazinones using 1 as catalyst. 
We examined this reaction in the absence of catalyst and it was found that when the reaction was carried out without any catalyst resulted in poor yield (table 1 , entry 1). The best result was obtained when 1 was used for which the yield was up to $90 \%$ (table 1 , entry 3 ). In addition, the result of this condensation in the presence of other silica immobilized acids (2-6) gave the corresponding product in slightly longer reaction time and lower yield (table 1, entries 5-9).

So, the optimal amount of 1 was $0.02 \mathrm{~g}\left(0.68 \mathrm{~mol} \%{ }^{6,8}\right.$ per $1 \mathrm{mmol}$ of $\beta$-naphthol at $150^{\circ} \mathrm{C}$ under solvent-free conditions. The lower loading amounts of $\mathbf{1}$ gave the corresponding product in lower yield and longer reaction time (table 1, entry 2). Therefore, we employed the optimized conditions $\left(0.02 \mathrm{~g} \mathrm{mmol}^{-1}\right.$ of 1 at $150^{\circ} \mathrm{C}$ under solvent-free conditions) for the condensation reaction of $\beta$-naphthol, urea with aromatic aldehydes into the corresponding naphthoxazinone derivatives (table 2).

A wide range of aromatic aldehydes were employed and all naphthoxazinones were obtained in good to high yields, demonstrating that this is a general method that tolerates bothelectron-withdrawing and electron-donating constituents. Aromatic aldehydes with electron-donating substituent such as $\mathrm{MeO}$, iso- $\mathrm{Pr}$, and $\mathrm{Me}$, were reacted with $\beta$-naphthol and urea under optimized conditions

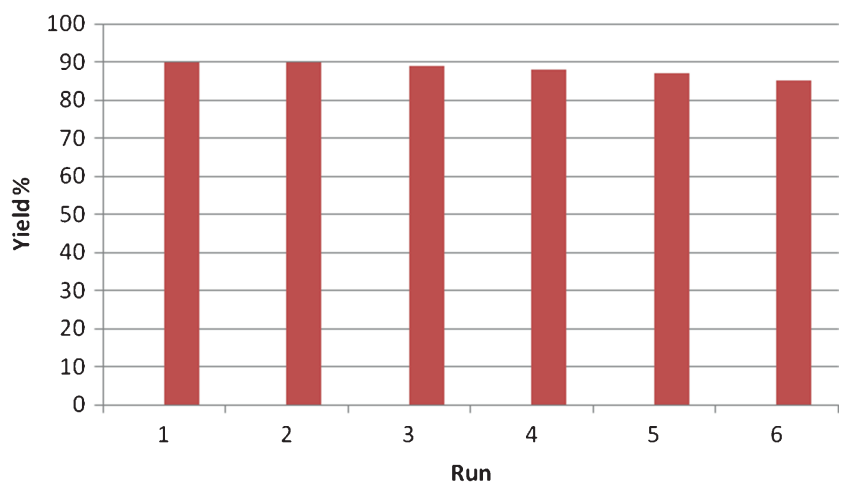

Figure 1. Recyclability of $\mathbf{1}(0.02 \mathrm{~g})$ in the reaction of $\beta$ naphthol $(1 \mathrm{mmol})$ with benzaldehyde $(1 \mathrm{mmol})$ and urea $(1.2 \mathrm{mmol})$ under solvent-free conditions at $150^{\circ} \mathrm{C}$. Time $=$ $60 \mathrm{~min}$. in good to high yield (table 2, entries 7-10). Aromatic aldehydes with electron-deficient such as 4-F, 3-F, and $3-\mathrm{NO}_{2}$ groups reacted with $\beta$-naphthol and urea under optimized conditions in high yield (table 2, entries 4-6). This method not only affords the products in good to high yield but also avoids the problems associated with catalyst cost, handling, safety, and pollution.

On the basis of the above observations and the literature reports, ${ }^{31,40}$ a plausible reaction pathway for the formation of naphthoxazinone is depicted in scheme 2 . The aryl aldehyde was protonated with solid acid $\mathbf{1}$ to generate the more electrophilic carbon center, followed by the nucleophilic attack of urea to give reactive acylimine intermediate. The resulting acylimine intermediate undergoes cyclization with 2-naphthol affording the corresponding desired naphthoxazinone followed by the elimination of ammonia.

The possibility of recycling the catalyst was examined using the reaction of benzaldehyde, $\beta$-naphthol and urea under optimized conditions. Upon completion, etha nol $(10 \mathrm{~mL})$ was added and the reaction mixture was filtered. The remaining solid was washed with warm ethanol, and the recycled catalyst was saved for the next reaction. The recycled catalyst could be reused five times without any further treatment. No observation of any appreciable loss in the catalytic activity of $\mathbf{1}$ was observed (figure 1).

Finally, a comparative study of $\mathbf{1}$ with other recently reported catalysts for the condensation of benzaldehyde and $\beta$-naphthol and urea as a model compound was made which revealed that $\mathbf{1}$ is an equally efficient and reusable catalyst (table 3 ).

\section{Conclusion}

In conclusion, we have shown that silica-bonded propyl-S-sulfonic acid (1), which can be prepared from commercially available and cheap starting materials, catalyzed efficiently this three-component condensation reaction for the synthesis of naphthoxazinones. The

Table 3. Comparison of the result of condensation reaction of benzaldehyde, $\beta$-naphthol and urea in the presence of different catalysts.

\begin{tabular}{|c|c|c|c|c|c|c|}
\hline Entry & Catalyst & Catalyst loading (g) & Conditions & Time (min) & Yield $(\%)^{\mathrm{a}}$ & Ref. \\
\hline 1 & $p$-TSA & $0.052(0.3 \mathrm{mmol})$ & Solvent-free $/ 160^{\circ} \mathrm{C}$ & 90 & 58 & 31 \\
\hline 2 & $\mathrm{TMSCl} / \mathrm{NaI}$ & (1.5 equiv.) & $\mathrm{DMF} / 140^{\circ} \mathrm{C}$ & 90 & 81 & 33 \\
\hline 3 & $\mathrm{HClO}_{4} / \mathrm{SiO}_{2}$ & $0.04(2 \mathrm{~mol} \%)$ & Solvent-free $/ 150^{\circ} \mathrm{C}$ & 360 & 88 & 34 \\
\hline 4 & $\mathrm{Cu}$ NPs & 0.001 & $\mathrm{~K}_{2} \mathrm{CO}_{3}, \mathrm{PEG} / \mathrm{rt}$ & 45 & 93 & 38 \\
\hline 5 & $\mathrm{ZnO}$ NPs & ( 0.3 equiv. $)$ & Solvent-free $/ 150^{\circ} \mathrm{C}$ & 90 & 85 & 40 \\
\hline 6 & $\mathrm{FeCl}_{3} / \mathrm{SiO}_{2} \mathrm{NPs}$ & $10-4(0.4 \mathrm{~mol} \%)$ & Solvent-free $/ 150^{\circ} \mathrm{C}$ & 10 & 85 & 41 \\
\hline 7 & 1 & $0.02(0.68 \mathrm{~mol} \%)$ & Solvent-free $/ 150^{\circ} \mathrm{C}$ & 60 & 90 & Present work \\
\hline
\end{tabular}

asolated Yield. 
simplicity of the eco-friendly and safe procedure, and reusability of catalyst are the advantages of this method.

\section{Supplementary Information}

Supplementary Information is available at www.ias.ac. in/chemsci.

\section{Acknowledgements}

We are thankful to Persian Gulf University Research Council for partial support of this work. Also, we are thankful to the School of Chemistry, Manchester University for NMR spectra.

\section{References}

1. Melero J A, Grieken R V and Morales G 2006 Chem. Rev. 1063790

2. Karimi B and Zareyee D 2008 Org. Lett. 103989

3. Niknam K and Saberi D 2009 Appl. Catal. A Gen. 366 220

4. Shakeri M S, Tajik H and Niknam K 2012 J. Chem. Sci. 1241025

5. Choudhary D, Paul S, Gupta R and Clark J H 2006 Green Chem. 8479

6. Niknam K, Saberi D and Nouri Sefat M 2009 Tetrahedron Lett. $\mathbf{5 0} 4058$

7. Niknam K and Saberi D 2009 Tetrahedron Lett. 50 5210

8. Niknam K, Saberi D and Baghernejad M 2009 Chin. Chem. Lett. 201444

9. Niknam K, Saberi D, Sadegheyan M and Deris A 2010 Tetrahedron Lett. 51692

10. Niknam K, Saberi D and Nouri Sefat M 2010 Tetrahedron Lett. $\mathbf{5 1} 2959$

11. Niknam K, Deris A, Naeimi F and Majleci F 2011 Tetrahedron Lett. 524642

12. Nouri Sefat M, Saberi D and Niknam K 2011 Catal. Lett. 1411713

13. Tayebi S, Baghernejad M, Saberi D and Niknam K 2011 Chin. J. Catal. 321477

14. Niknam K, Jafarpour N and Niknam E 2011 Chin. Chem. Lett. 2269

15. Iravani N, Mohammadzade N S and Niknam K 2011 Chin. Chem. Lett. 221151

16. Rahi T, Baghernejad M and Niknam K 2012 Chin. J. Catal. 331095
17. Niknam K, Jamali A, Tajaddod M and Deris A 2012 Chin. J. Catal. 331312

18. Tavakoli Z, Baghernejad M and Niknam K $2012 \mathrm{~J}$. Heterocycl. Chem. 49634

19. Baghernejad M and Niknam K 2012 Int. J. Chem. 4 52

20. Pargaleh Brojeni S, Baghernejad M, Saberi D and Niknam K 2013 Green Chem. Lett. Rev. 669

21. Niknam K and Piran A 2013 Green Sustainable Chem. 32 A 1

22. Ghasemi S, Baghernejad M and Niknam K 2013 Iran. J. Catal. 3165

23. Niknam K, Zolfigol M A, Saberi D and Molaee H 2009 J. Chin. Chem. Soc. 561257

24. Niknam K, Hasaninejad A and Arman M 2010 Chin. Chem. Lett. 21399

25. Waxman L and Darke P L 2000 Antiviral Chem. Chemother. 111

26. Latif N, Mishriky N and Assad F M 1982 Aust. J. Chem. 351037

27. Wang Y, Li X and Ding K 2002 Tetrahedron Asymmet. 131291

28. Holly F W and Cope A C 1944 J. Am. Chem. Soc. 66 1875

29. Szatmari I, Hetenyi A, Lazar L and Fulop F 2004 J. Heterocycl. Chem. 41367

30. Cimarelh C, Palmieri G and Volpini E 2004 Can. J. Chem. 821314

31. Dabiri M, Delbari A S and Bazgir A 2007 Synlett 821

32. Dabiri M, Delbari A S and Bazgir A 2007 Heterocycles 71543

33. Sabitha G, Arundhathi K, Sudhakar K and Sastry B S 2010 J. Heterocycl. Chem. 47272

34. Abbastabar Ahangar H, Mahdavinia G H, Marjani $\mathrm{K}$ and Hafezian A 2010 J. Iran. Chem. Soc. 7770

35. Chenggang J, Xin G, Zonglei Zh, Hangxian X and Cunde W 2010 J. Chem. Res. 3419

36. Kottawar S S, Siddiqui S A and Bhusare S R 2010 RASAYAN J. Chem. 3646

37. Chaskar A, Vayavhare V, Padalkar V, Phatangare K and Deokar H 2011 J. Serb. Chem. Soc. 7621

38. Kumar A, Saxena A, Dewan M, De A and Mozumdar S 2011 Tetrahedron Lett. 524835

39. Lei M, Ma L and Hu L 2011 Synth. Commun. 413424

40. Rao D G B, Kaushik M P and Halve A K 2012 Tetrahedron Lett. 532741

41. Safaei-Ghomi J, Zahedi S and Ghasemzadeh M A 2012 Iran. J. Catal. 227

42. GharibA Khorasani B R H Jahangir M and Roshani M 2013 Bulgarian Chem. Commun. 4559 